\title{
Evaluation of Pleural-Based Nodules in a 52-Year-Old Woman with a History of Metastatic Breast Cancer
}

Blaine T. Mischen and Marques L. Bradshaw

Nuclear Medicine, Radiology Department, Medical University of South Carolina, Charleston, South Carolina

\begin{abstract}
A
52-y-old woman with a history of invasive ductal carcinoma had undergone right mastectomy, right axillary lymph node dissection, and breast reconstruction. Two of six axillary lymph nodes were positive for ductal carcinoma at surgery, and the patient was staged pT2 pN1a Mx. A one-month follow-up chest CT scan showed multiple left-sided pleural-based nodules suggestive of metastatic disease. PET/CT, which was performed to further characterize these nodules, did not demonstrate significant ${ }^{18} \mathrm{~F}$-FDG uptake within them or evidence of metastatic disease (Fig. 1). In the process of formulating a differential diagnosis for these nodules, it was discovered that, as a child, the patient had sustained an accidental gunshot wound. The possibility of thoracic splenosis was suggested, and a ${ }^{99 \mathrm{~m}} \mathrm{Tc}$-labeled heat-denatured red blood cell (DRBC) scan was ordered to confirm the origin of these conspicuous pleural-based nodules.
\end{abstract}
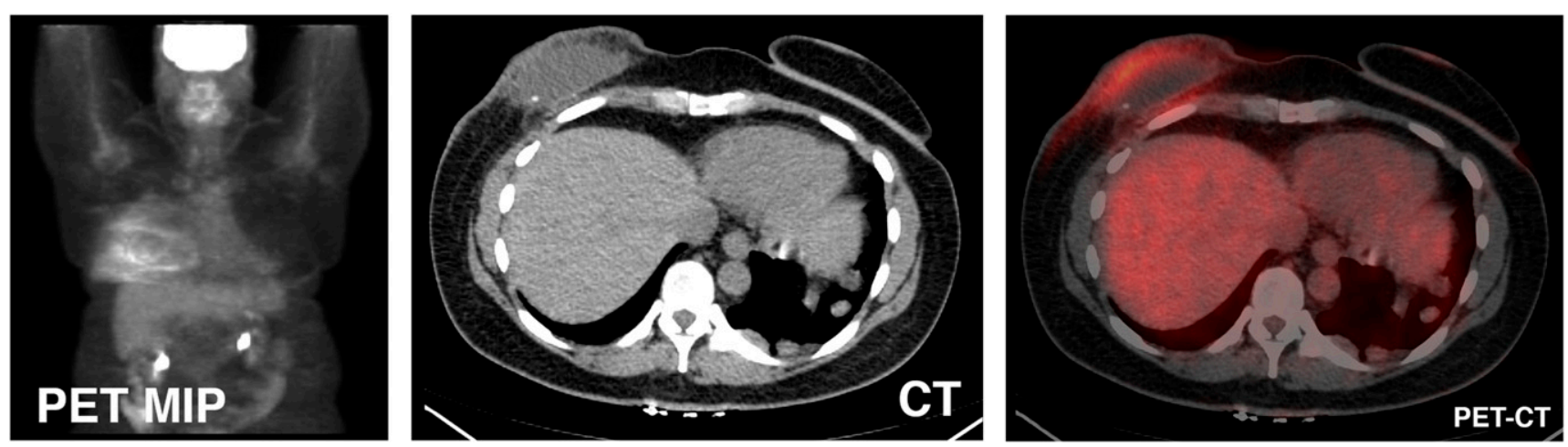

FIGURE 1. Initial maximum-intensity-projection (MIP) PET, CT, and PET/CT images.

A blood sample was drawn into a vial containing anticoagulant (acid citrate dextrose) labeled with $370 \mathrm{MBq}(10 \mathrm{mCi})$ of ${ }^{99 \mathrm{~m}} \mathrm{Tc}$-pertechnetate and heat-denatured by submersion in a $49.5^{\circ} \mathrm{C}\left( \pm 0.5^{\circ} \mathrm{C}\right)$ water bath for 20 min. Standard quality control analyses were completed. The radiolabeled autologous DRBCs were reinjected intravenously. Planar anterior and posterior images of the chest and abdomen were obtained 30 min after radiolabeled DRBC reinjection. Congruent SPECT and CT images of the chest and abdomen were also acquired and were displayed with and without fusion.

\section{QUESTION 1}

Localization of DRBCs takes place by what mechanism?

A. Active transport by splenocytes via the adenosine triphosphatase-driven Na-K pump.

B. Binding to transferrins, which localize to splenic tissue.

C. Ion exchange on the cell membrane of splenocytes.

D. Phagocytosis of the DRBCs by the reticuloendothelial system and size- and shape-dependent sequestration by the spleen. 


\section{QUESTION 2}

Regarding the sensitivity and specificity of different imaging modalities to evaluate for splenosis, which statement is true?

A. ${ }^{99 m}$ Tc-DRBC scans have a higher sensitivity and specificity than sulfur colloid, CT, and MRI.

B. ${ }^{99 \mathrm{~m}} \mathrm{Tc}-\mathrm{DRBC}$ scans and sulfur colloid scans are equal in sensitivity and specificity, and both are superior to CT and MRI.

C. MRI is the gold standard in evaluating splenosis, having the highest sensitivity and specificity when compared with all other imaging modalities.

D. All imaging modalities are the same with regard to the sensitivity and specificity of splenosis detection.

\section{CASE DISCUSSION}

Given the patient's history of locally metastatic breast cancer, the pleural-based nodules seen on CT were suspected of being new metastatic disease; however, the non- ${ }^{18}$ F-FDG avidity of these nodules was a confounding result (Fig. 1). To formulate a more extensive differential diagnosis, additional past medical history was sought, and it was discovered the patient had sustained an accidental gunshot wound as a child. The trajectory of the bullet was from the left posterior chest wall to the diaphragm, spleen, and bowel and exiting through the anterior medial abdominal wall. This trauma required surgical repair of the bowel injuries, as well as a splenectomy.

The autotransplantation of splenic tissue to the thoracic cavity requires particular circumstances. Specifically, splenic trauma or surgery, violation of the diaphragm, and an appropriate seeding environment are necessary. Therefore, thoracic splenosis is a rare and usually incidental finding on various imaging studies (3). Differentiating splenosis from other, more common, pleural-based nodules, particularly when remote from the traumatic seeding event or without a pertinent past medical history, can be quite difficult. The diagnosis is further complicated when the patient has a known neoplasm, as in this case. However, if splenosis is suspected, the use of a noninvasive test with a high sensitivity and specificity can decrease radiation exposure from further imaging, avoid unnecessary biopsies and their associated sequelae, and give the patient and the clinician assurance of a definitive diagnosis. ${ }^{99} \mathrm{~m}$ Tc-DRBC studies are highly sensitive at detecting even small volumes of splenic tissue and are very specific $(2,4)$. Additionally, there is a higher target (splenic tissue) to background ratio in ${ }^{99 m}$ Tc-DRBC scans than in radiolabeled sulfur colloid scans due to the marked splenic reticuloendothelial system phagocytosis and pulp sequestration of damaged red blood cells $(\leq 50 \%)$ compared with colloid distribution (10\%-15\% spleen, $80 \%-85 \%$ liver, and $5 \%$ bone marrow) $(5,6)$.
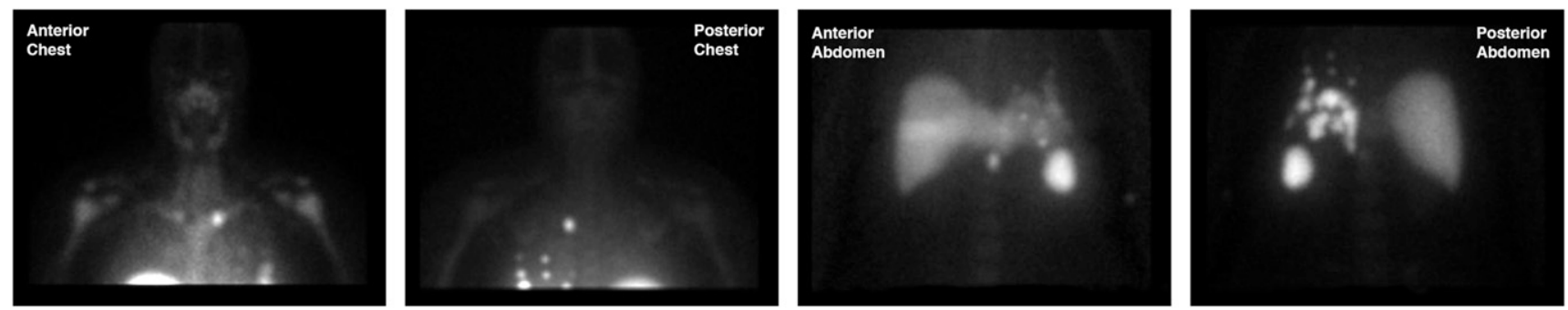

FIGURE 2. Planar 99mTc-labeled DRBC images showing numerous foci of uptake.
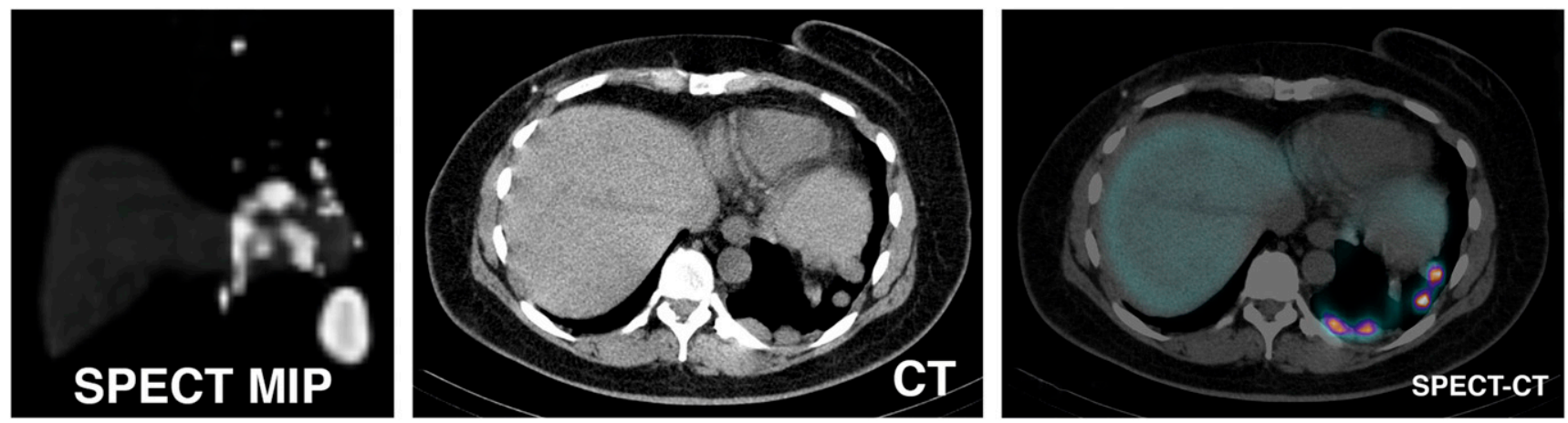

FIGURE 3. Maximum-intensity-projection (MIP) SPECT, CT, and SPECT/CT images confirming activity in all previously seen pleural-based nodules. 

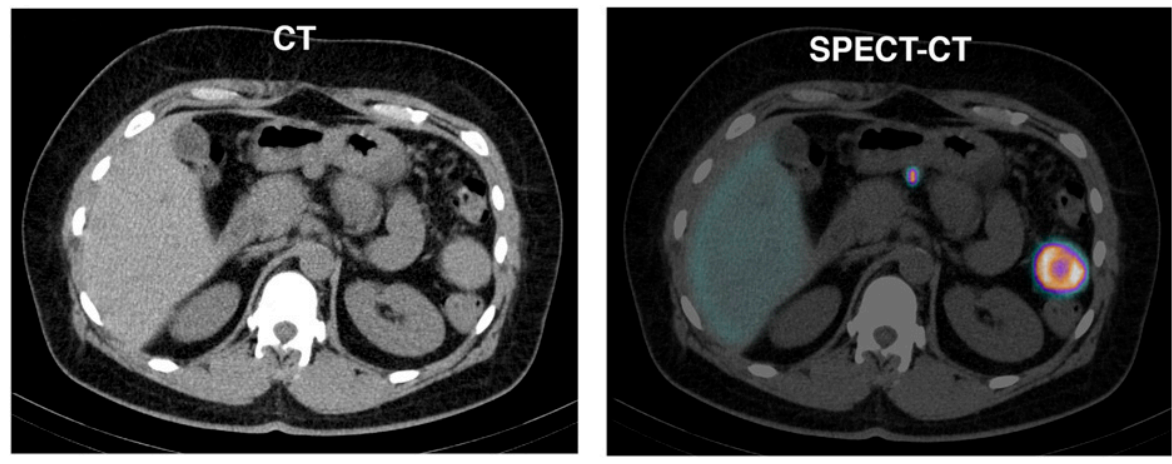

FIGURE 4. CT and SPECT/CT images showing two islands of peritoneal splenosis.

In this case, numerous foci of ${ }^{99 \mathrm{~m} T c-D R B C s}$ were detected on the planar images (Fig. 2). Hybrid SPECT/CT images confirmed significant ${ }^{99} \mathrm{~m} \mathrm{Tc}-\mathrm{DRBC}$ activity in all previously seen pleural-based nodules, thus supporting the diagnosis of thoracic splenosis (Fig. 3). Additionally, two islands of peritoneal splenosis were localized (Fig. 4).

\section{REFERENCES}

1. Stewart CA, Sakimura ME. Scintigraphic demonstration of splenosis. Clin Nucl Med. 1986;11:161-164.

2. Armas RR. Clinical studies with spleen-specific radiolabeled agents. Semin Nucl Med. 1985;15:260-275.

3. Hagman TF, Winer-Muram HT, Meyer CA, Jennings SG. Intrathoracic splenosis: superiority of technetium Tc 99m heat-damaged RBC imaging. Chest. 2001;120:20972098.

4. Yammine JN, Yatim A, Barbari A. Radionuclide imaging in thoracic splenosis and a review of the literature. Clin Nucl Med. 2003;28:121-123.

5. Atkins HL, Goldman AG, Fairchild RG, et al. Splenic sequestration of ${ }^{99 \mathrm{~m}} \mathrm{Tc}$ labeled, heat treated red blood cells. Radiology. 1980;136:501-503.

6. Massey MD, Stevens JS. Residual spleen found on denatured red blood cell scan following negative colloid scans. J Nucl Med. 1991;32:2286-2287.

${ }^{\star}$ For the answers, see page 17A. 